\title{
A novel AMPK activator hernandezine inhibits LPS-induced TNFa production
}

\author{
Ping Li ${ }^{1, *}$, Xiaofang $\mathbf{L i}^{2, *}$, Yonghong $\mathbf{W u}^{3}$, Manxiang $\mathbf{L i}^{4}$ and Xiaochuang Wang \\ ${ }^{1}$ Department of Emergency, The Second Affiliated Hospital of Xi'an Jiao Tong University, Xi'an, China \\ ${ }^{2}$ Department of Gastroenterology, The Third People's Hospital of Xi'an, Xi'an, China \\ ${ }^{3}$ Staff Room of Clinical Immunology and Pathogen Detection, Medical Technology Department, Xi'an Medical College, Xi'an, \\ China \\ ${ }^{4}$ Department of Respiratory Medicine, The First Affiliated Hospital of Xi'an Jiao Tong University, Xi'an, China \\ ${ }^{5}$ Department of Critical Care Medicine, The Second Affiliated Hospital of Xi'an Jiao Tong University, Xi'an, China \\ *These authors have contributed equally to this work \\ Correspondence to: Xiaochuang Wang, email: wangxiaochuangyshi@126.com \\ Manxiang Li, email: drli_manxiang88@163.com \\ Keywords: hernandezine, LPS, AMPK, TNFa, NFKB \\ Received: March 08, $2017 \quad$ Accepted: May 03, $2017 \quad$ Published: June 05, 2017 \\ Copyright: Li et al. This is an open-access article distributed under the terms of the Creative Commons Attribution License 3.0 (CC BY 3.0), \\ which permits unrestricted use, distribution, and reproduction in any medium, provided the original author and source are credited.
}

\section{ABSTRACT}

Here, we found that hernandezine, a novel AMPK activator, inhibited LPS-induced TNFa expression/production in human macrophage cells (THP-1 and U937 lines). Activation of AMPK is required for hernandezine-induced anti-LPS response. AMPKa shRNA or dominant negative mutation (T172A) blocked hernandezine-induced AMPK activation, which almost completely reversed anti-LPS activity by hernandezine. Exogenous expression of the constitutively activate AMPKa (T172D, caAMPKa) also suppressed TNFa production by LPS. Remarkably, hernandezine was unable to further inhibit LPS-mediated TNFa production in caAMPKa-expressing cells. Hernandezine inhibited LPS-induced reactive oxygen species (ROS) production and nuclear factor kappa B (NFKB) activation. Treatment of hernandezine in ex-vivo cultured primary human peripheral blood mononuclear cells (PBMCs) also largely attenuated LPSinduced TNFa production. Together, we conclude that AMPK activation by hernandezine inhibits LPS-induced TNFa production in macrophages/monocytes.

\section{INTRODUCTION}

Patients with chronic obstructive pulmonary disease (COPD) often suffer chronic yet persistent airway inflammations [1-3]. Many pathogen-associated molecular patterns (PAMPs) are circulating in lungs of the COPD patients [1-3]. Lipopolysaccharide (LPS) is one of the most prominent PAMPs $[4,5]$. LPS activates resident monocytes/macrophages to produce TNF $\alpha$ (tumor necrosis factor- $\alpha$ ) and other pro-inflammatory cytokines $[4,5]$. $\mathrm{TNF} \alpha$ level is significantly elevated in bronchoalveolar lavage fluids, sputum, as well as plasma and lung of COPD patients [6-8]. Anti-TNF $\alpha$ strategy could efficiently lessen COPD patients' inflammations [6-8]. The research focus of our group is to explore the underlying mechanisms of
LPS-induced TNF $\alpha$ production, which could possibly help to develop intervention agents [9-11].

AMP-activate protein kinase (AMPK) is the key sensor of cellular energy status [12, 13]. Evidences (including ours [9-11]) have implied a key function of AMPK in suppressing inflammatory responses [14-17]. Several AMPK activators, including AICAR, A769662 and GSK621, significantly attenuated LPS-mediated nuclear factor kappa $\mathrm{B}(\mathrm{NF \kappa B})$ activation and cytokine production [10, 14, 15]. Metformin, another AMPK activator, attenuated expression of pro-inflammatory and adhesion molecule [18]. Further, perifosine activated AMPK signaling and inhibited LPS-induced TNFa production [15]. Cordycepin-activated AMPK also significantly inhibited TNF $\alpha$ expression by LPS [19]. 
Thus, AMPK activation represents a novel and efficient strategy to inhibit LPS inflammatory response $[10,11,14$, $15,18,19]$.

Hernandezine is an alkaloid isolated from Chinese medicinal herb manyleaf meadowure rhizome and root [20]. A very recent study by Law et al., has characterized hernandezine as a novel AMPK activator [20]. In the current report, we show that hernandezine inhibits LPSinduced TNF $\alpha$ production via activating AMPK signaling.

\section{RESULTS}

\section{The effect of hernandezine on macrophage cell survival and $\mathrm{TNF} \alpha$ production}

First, we tested the potential effect of hernandezine on the survival of human macrophage cells. U937 cells (macrophage cell line [11]) were treated with gradually increased concentrations of hernandezine (1-100 $\mu \mathrm{M})$ for 24 hours, trypan blue staining assay [11] was applied to test cell survival. Viable cells were trypan blue negative [11]. Results in Figure 1A demonstrated that hernandezine was not cytotoxic to U937 cells until at $100 \mu \mathrm{M}$, the latter induced obvious U937 cell death (Figure 1A). Histone
DNA apoptosis ELISA assay [10,11] results in Figure 1B demonstrated that only $100 \mu \mathrm{M}$ of hernandezine induced significant U937 cell apoptosis. It was not pro-apoptotic at lower concentrations (Figure 1B). As shown in Figure $1 \mathrm{C}$, treatment with hernandezine (1-30 $\mu \mathrm{M}$, non-cytotoxic concentrations) failed to change basal TNF $\alpha$ production in U937 cells. However, at $100 \mu \mathrm{M}$, hernandezine inhibited TNF $\alpha$ production (Figure 1C), which could be probably due to cell death (Figure 1A and 1B). The similar experiments were also performed in the other human macrophage cell line: THP-1 [11]. Results showed that hernandezine was indeed not cytotoxic (Figure 1D) nor pro-apoptotic (Figure 1E) to THP-1 cells until at a high concentration $(100 \mu \mathrm{M})$. Basal TNF $\alpha$ production in THP-1 cells was also not changed in hernandezine-treated THP-1 cells, except at $100 \mu \mathrm{M}$ (Figure 1F).

\section{The effect of hernandezine on LPS-induced TNF $\alpha$ production in macrophage cells}

One main focus of this study is to test the potential effect of hernandezine on LPS-induced proinflammatory activity. In line with our previous findings [11], treatment with LPS (100 $\mathrm{ng} / \mathrm{mL})$ induced dramatic $\mathrm{TNF} \alpha$ mRNA expression (Figure 2A) and protein

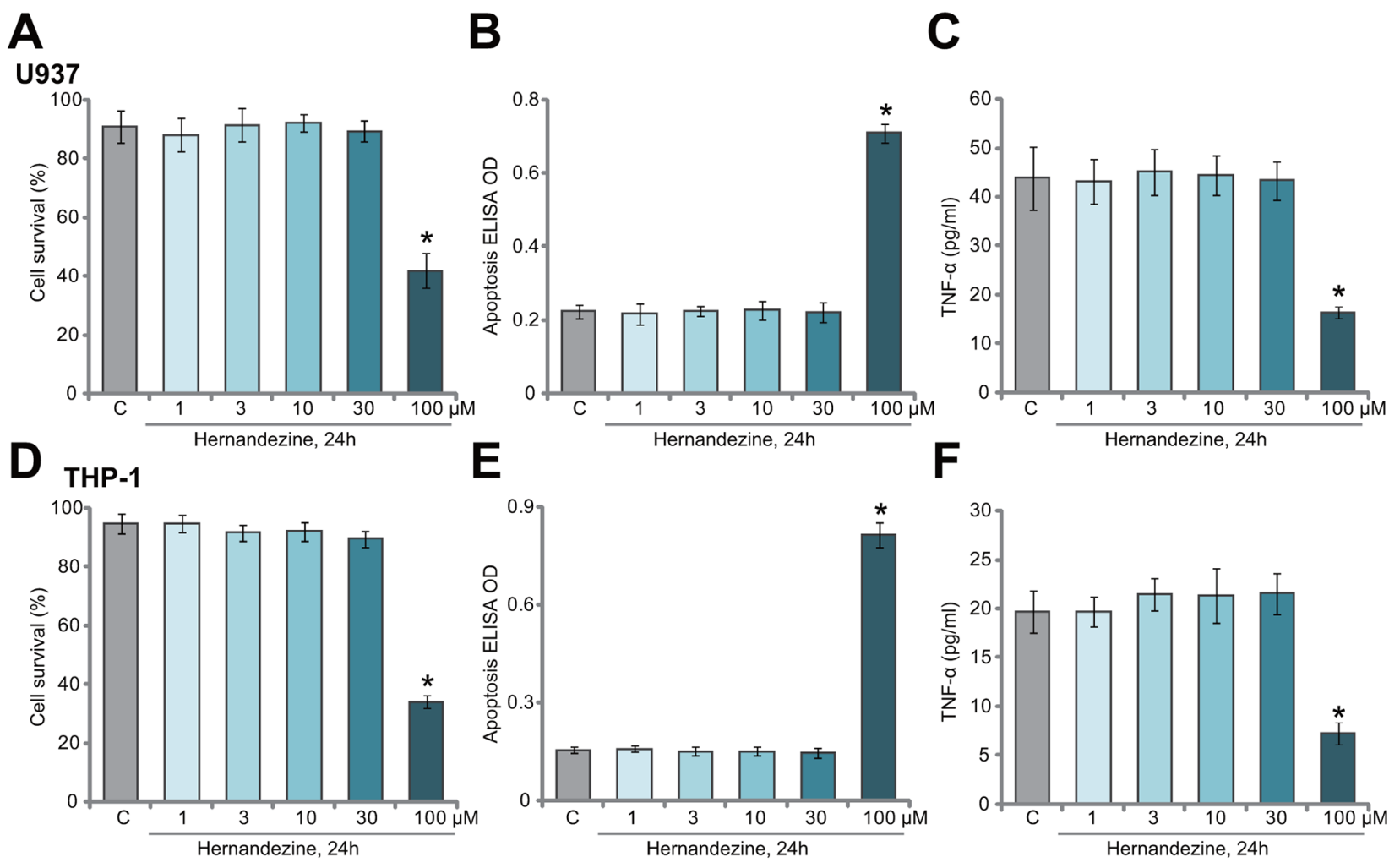

Figure 1: The effect of hernandezine on macrophage cell survival and TNFa production. Human macrophage cells, U937 line (A-C) and THP-1 line (D-E), were either left untreated ("C", same for all figures) or treated with hernandezine (1-100 $\mu \mathrm{M})$, cells were further cultured for 24 hours, cells were further cultured for 24 hours, cell survival (A and D, trypan blue assay), apoptosis (B and E, Histone DNA ELISA assay) and medium TNF $\alpha$ content (C and F, ELISA assay) were tested. ${ }^{*} p<0.05 v s$. "C". Experiments in this figure were repeated three times, and similar results were obtained. 
secretion (Figure 2B) in U937 cells. Remarkably, co-treatment with hernandezine (at 10 and $30 \mu \mathrm{M}$ ), significantly attenuated LPS-induced TNF $\alpha$ expression and production (Figure 2A and $2 \mathrm{~B}$ ). Hernandezine demonstrated a dose-dependent response in inhibiting TNFa production (Figure 2A and 2B). At lower concentrations ( 1 and $3 \mu \mathrm{M})$, hernandezine was ineffective on LPS (Figure 2A and 2B). Notably, similar results were also obtained in THP-1 macrophage cells, where hernandezine $(10 / 30 \mu \mathrm{M})$ largely attenuated LPS (100 ng/mL)-induced TNF $\alpha$ mRNA expression (Figure 2C) and production (Figure 2D). Once again, hernandezine at $1 / 3 \mu \mathrm{M}$ was ineffective in THP-1 cells (Figure 2C and 2D). These results demonstrate that hernandezine inhibits LPS-induced TNF $\alpha$ production in macrophage cells.

\section{Activation of AMPK is required for hernandezine-induced anti-LPS response}

Our group [10, 11] and others [15, 16, 19] have implied that activation of AMPK could inhibit LPS- induced pro-inflammatory response. The study by Law et al., has confirmed that hernandezine is a novel AMPK activator [20]. We therefore tested AMPK signaling in hernandezine-treated macrophage cells. As shown in Figure 3A, hernandezine dose-dependently induced AMPK activation in U937 cells. As phosphorylated("p-") AMPK $\alpha$ and p-acetyl-CoA carboxylase (p-ACC, the main downstream target protein of AMPK $[12,21])$ were significantly increased after treatment of 10 and 30 $\mu \mathrm{M}$ of hernandezine (See quantified blot results in Figure $3 \mathrm{~A})$. On the other hand, $1 \mu \mathrm{M}$ and $3 \mu \mathrm{M}$ of hernandezine failed to induce significant AMPK activation (See quantified blot results in Figure 3A). Total AMPK $\alpha$ and ACC expression was unchanged following hernandezine treatment.

To study the link between AMPK activation and hernandezine-induced anti-LPS response, shRNA method was utilized to knockdown AMPK $\alpha$. In line with our previous studies $[10,11]$, two AMPK $\alpha$ shRNAs with nonoverlapping sequences were applied. The two shRNAs were named as "shAMPK $\alpha-N o .1$ " and "shAMPK $\alpha$ No.2" $[10,11]$. AMPK $\alpha$ was indeed silenced in U937
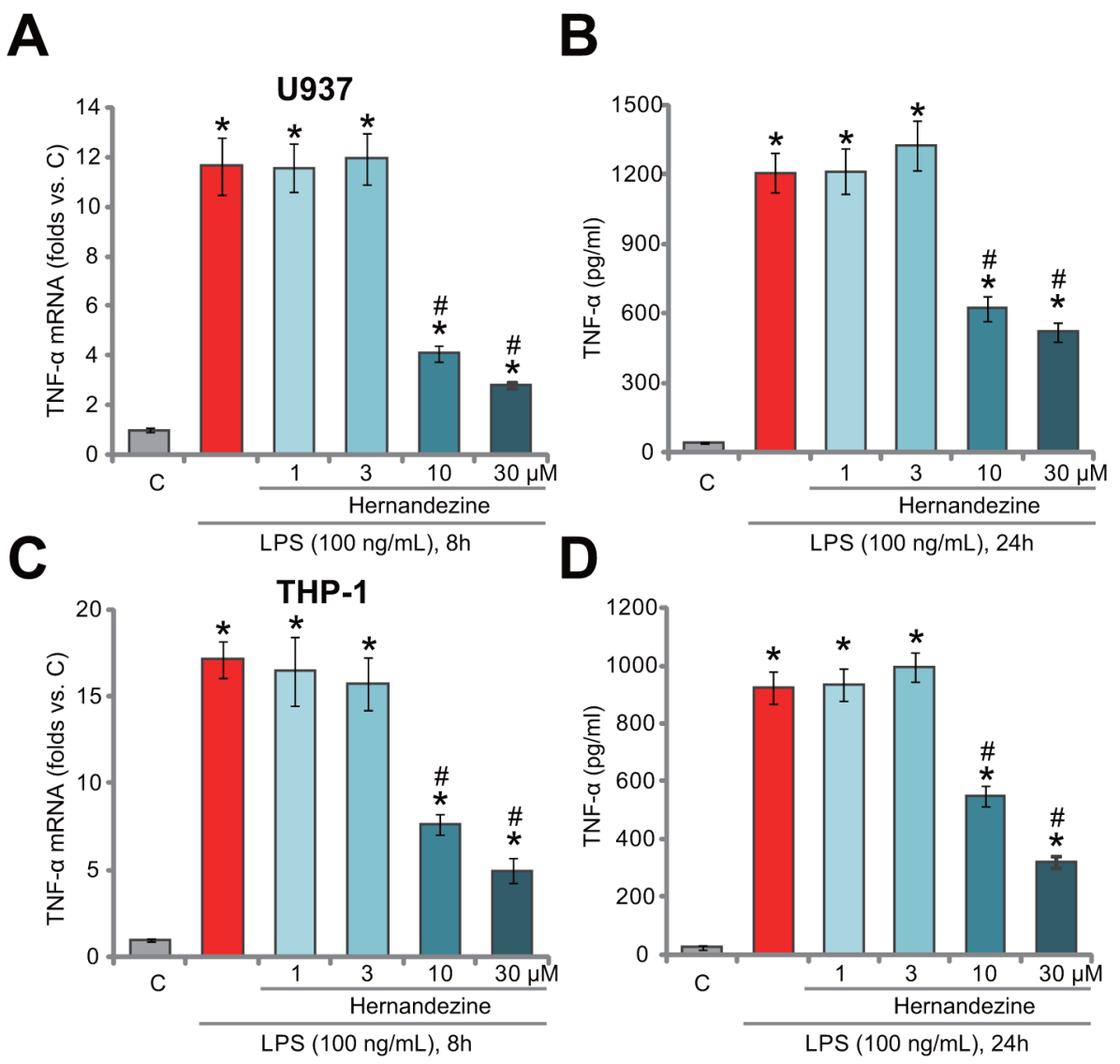

Figure 2: The effect of hernandezine on LPS-induced TNFa production in macrophage cells. Human macrophage cells, U937 line (A and B) and THP-1 line (C and D), were treated with LPS (100 ng/mL) or plus hernandezine (1-30 $\mu \mathrm{M})$, cells were further cultured in the conditional medium for indicated time, TNF $\alpha$ mRNA expression (A and $\mathbf{C}$ ) and TNF $\alpha$ protein content (in conditional medium, B and D) were tested by qRT-PCR assay and ELISA assay, respectively. ${ }^{*} p<0.05 v s$. "C". " $p<0.05 v s$. LPS only treatment. Experiments in this figure were repeated three times, and similar results were obtained. 
cells expressing the AMPK $\alpha$ shRNA (See quantified blot results in Figure 3B). Consequently, hernandezineinduced AMPK activation, or p-AMPK $\alpha$, was almost blocked (See quantified blot results in Figure 3B). AMPK $\alpha$ shRNAs didn't change LPS-induced TNFa mRNA expression (Figure 3C) and production (Figure 3D) in U937 cells. Remarkably, in AMPK $\alpha$-silenced U937 cells, hernandezine-induced anti-LPS response was largely compromised (Figure 3C and 3D). In another words, hernandezine was unable to inhibit LPS-induced TNF $\alpha$ synthesis (Figure 3C) and production (Figure 3D) when AMPK was silenced. These results imply that activation of AMPK is required for hernandezine-induced anti-LPS response.

\section{AMPKa dominant negative mutation abolishes hernandezine-induced anti-LPS response}

To further support the requirement of AMPK activation in hernandezine-induced anti-LPS response, a dominant negative mutant AMPK $\alpha$ (T172A, "dnAMPK $\alpha$ ") [11, 22-24] was introduced to U937 cells. Western blotting assay results in Figure 4A confirmed the expression of the dnAMPK $\alpha$ in the stable U937 cells. Notably, hernandezine-induced AMPK activation, or p-AMPK $\alpha$ / ACC, was almost blocked in dnAMPK $\alpha$-expression U937 cells (Figure 4A). Consequently, hernandezine-induced anti-LPS response was significantly attenuated (Figure 4B and 4C). Hernandezine was largely ineffective against LPS-induced TNF $\alpha$ mRNA expression (Figure 4B) and production (Figure 4C) when AMPK was mutant. Next, the constitutively-activate AMPK $\alpha$ (T172D, "caAMPK $\alpha$ ") $[10,23]$ was introduced to U937 cells. Stable cells with caAMPK $\alpha$ were again established. As demonstrated, LPS-induced TNF $\alpha$ mRNA expression (Figure 4D) and production (Figure 4E) were largely attenuated in the caAMPK $\alpha$-expressing U937 cells. Remarkably, the antiLPS activity of hernandezine was nullified in caAMPK $\alpha$ expressing cells (Figure 4D and 4E). Hernandezine was unable to further suppress LPS-induced TNF $\alpha$ production
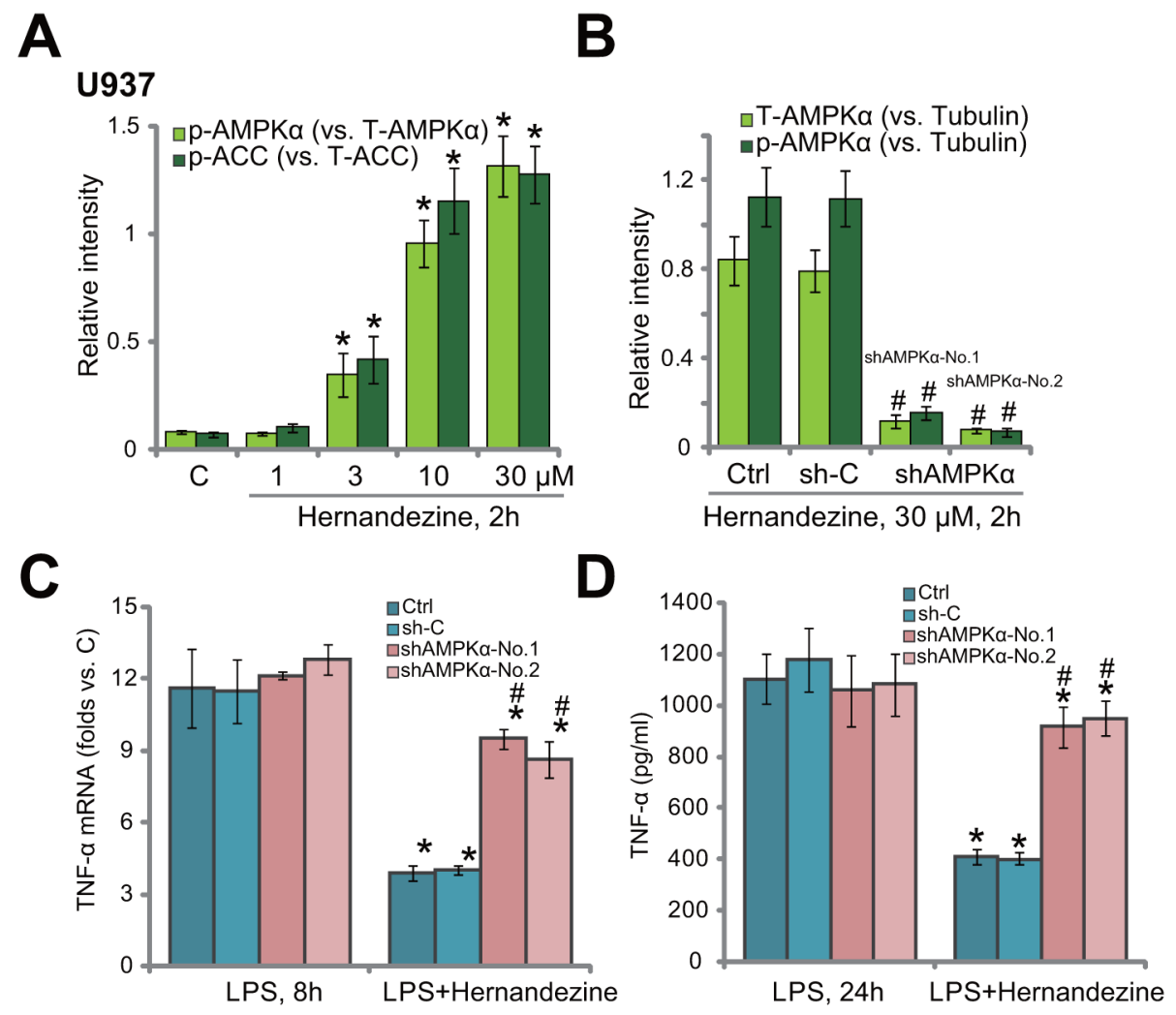

Figure 3: Activation of AMPK is required for hernandezine-induced anti-LPS response. U937 cells were treated with hernandezine (1-30 $\mu \mathrm{M})$ for 2 hours, expression of listed proteins was tested by Western blotting assay, and blot data of three sets of repeat were quantified (A) U937 cells were infected with lentiviral AMPK $\alpha$ shRNA ("shAMPK $\alpha$-No.1"/"shAMPK $\alpha$-No.2") or scramble control shRNA ("sh-C"), and stable cells were established; cells were treated with hernandezine $(30 \mu \mathrm{M})$ for 2 hours, expression of listed proteins was tested by Western blotting assay (blot data of three sets of repeat were quantified (B)); cells were also treated with LPS (100 ng/mL), $\mathrm{TNF} \alpha \mathrm{mRNA}$ expression (C) and protein content (in conditional medium, (D)) were tested. "Ctrl" stands for un-infected cells. *p $<0.05$ vs. "C" (A). \# p<0.05 vs. "sh-C" group (B). *p<0.05 vs. LPS only group (C and D). \# p $<0.05$ vs. hernandezine of "sh-C" group (C and D). Experiments in this figure were repeated three times, and similar results were obtained. 
when AMPK was already constitutively-activated (Figure 4D and 4E). These results again confirmed that activation of AMPK is required for hernandezine-induced anti-LPS activity.

\section{Hernandezine inhibits LPS-induced ROS production and NF-kB activation}

As discussed, forced-activation of AMPK was shown to efficiently suppress LPS-induced ROS production and subsequent nuclear factor $\mathrm{kB}(\mathrm{NF}-\mathrm{kB})$ activation $[10,11$, $15,16,19]$, leading to $\mathrm{TNF} \alpha$ transcription inhibition in monocytes/macrophages. Here, we showed that treatment with hernandezine $(30 \mu \mathrm{M})$ in U937 cells largely attenuated LPS-induced ROS production (Figure 5A). Further, NF-kB activation in LPS-treated U937 cells was also inhibited by hernandezine (Figure 5B). Importantly, AMPK $\alpha$ knockdown (by targeted shRNA, shAMPK $\alpha$-No.1) or mutation (by expressing $\operatorname{dn} \mathrm{AMPK} \alpha$ ) almost abolished hernandezine- induced inhibition on ROS and NF-kB (Figure 5A and 5B). These results suggest that AMPK activation is required for hernandezine-induced inhibition on ROS production and NF-kB activation in LPS-treated cells.

\section{Hernandezine inhibits LPS-induced TNFa production in primary human peripheral blood mononuclear cells (PBMCs)}

At last, we tested the potential activity of hernandezine in human monocytes. In consistent with our previous studies $[9,10]$, primary PBMCs from COPD patients were ex-vivo cultured. Trypan blue assay results in Figure 6A showed again that treatment with $30 \mu \mathrm{M}$ of hernandezine (or plus LPS) was non-cytotoxic to the primary PBMCs. Significantly, hernandezine remarkably inhibited LPS-induced TNF $\alpha$ mRNA expression (Figure $6 \mathrm{~B}$ ) and production (Figure 6C). Therefore, in line with the cell line data, hernandezine similarly inhibits LPS-
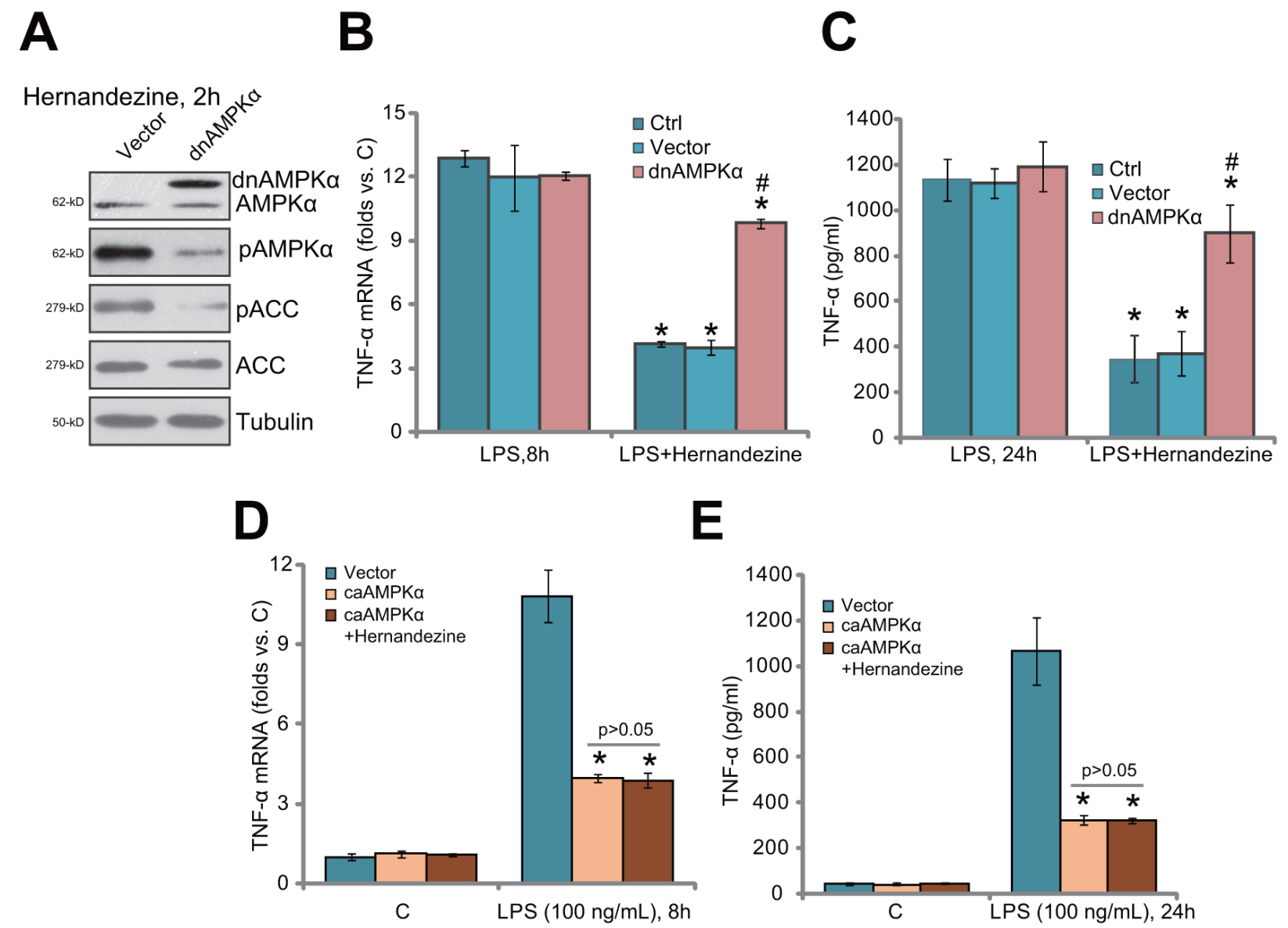

Figure 4: AMPKa dominant negative mutation abolishes hernandezine-induced anti-LPS response. U937 cells were constructed with the dominant negative mutant AMPK $\alpha$ (T172A, "dnAMPK $\alpha$ "), the constitutively activate AMPK $\alpha$ (T172D, "caAMPK $\alpha$ ") or the empty vector (pSuper-puro, "Vec"), and stable cells were established; Cells were treated with hernandezine (30 $\mu$ M) or plus LPS (100 $\mathrm{ng} / \mathrm{mL}$ ) for indicated time, expression of listed proteins was tested by Western blotting assay (A); TNF $\alpha$ mRNA expression (B and D) and protein content (in conditional medium, (C and $\mathbf{E}$ ) were tested by qRT-PCR assay and ELISA assay, respectively. ${ }^{*} p<0.05 v s$. LPS only group. ${ }^{*} p<0.05$ vs. hernandezine of "Vec" group. Experiments in this figure were repeated three times, and similar results were obtained. 
induced TNF $\alpha$ production and expression in primary human PBMCs.

\section{DISCUSSION}

We have previously shown that GSK621, the novel AMPK activator [25], attenuated LPS-induced $\mathrm{TNF} \alpha$ production [10]. Similarly, our very recent study has demonstrated that microRNA-135b-5p ("miR- 135b-5p") inhibited LPS-induced TNF $\alpha$ production via activating AMPK [11]. miR-135b-5p activated AMPK signaling via silencing its phosphatase Ppm1e [11]. These results indicate that AMPK activation could be a novel and efficient strategy to inhibit LPS-induced pro-inflammatory response. Here, we showed that AMPK activation is also required for hernandezinemediated anti-LPS response. AMPK knockdown (by targeted shRNAs) or dominant negative mutation
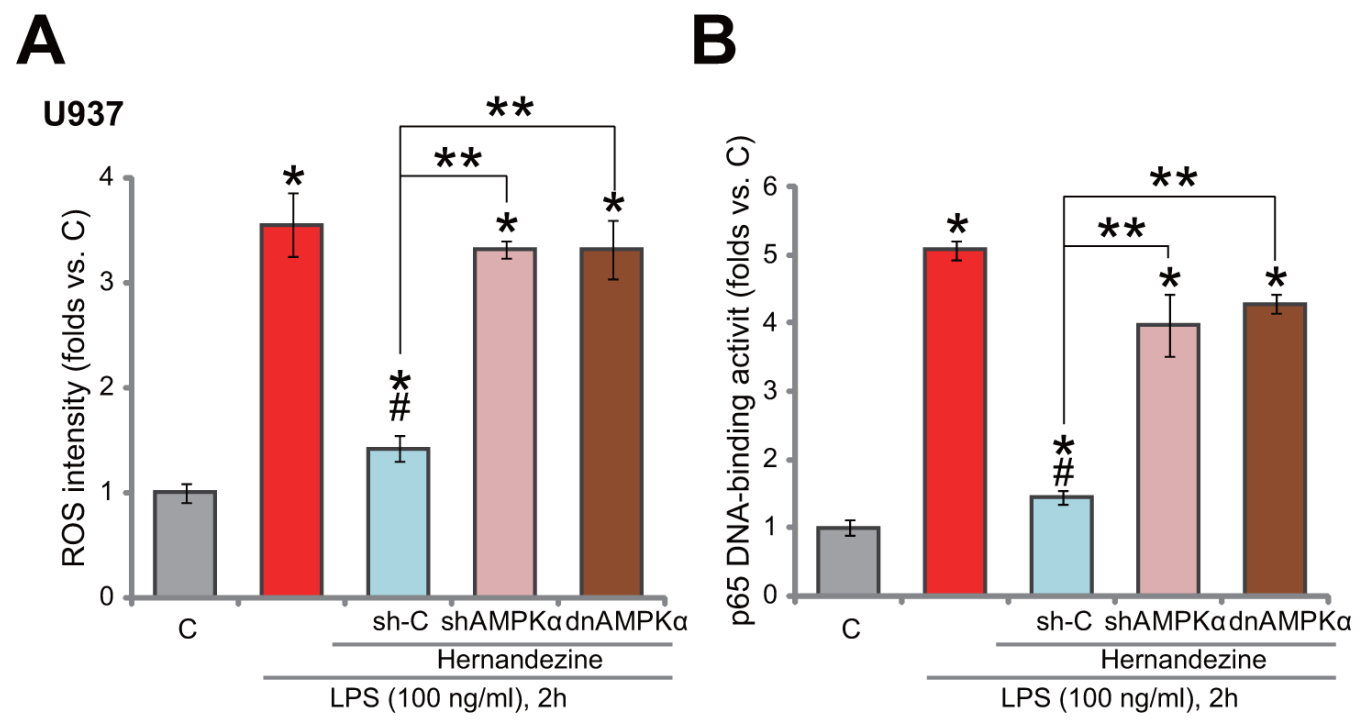

Figure 5: Hernandezine inhibits LPS-induced ROS production and NF-kB activation. Stable U937 cells, with scramble control shRNA ("sh-C"), AMPK $\alpha$ shRNA ("shAMPK $\alpha$ ", No.1) or dominant negative AMPK $\alpha$ (T172A, "dnAMPK $\alpha$ "), were treated with LPS $(100 \mathrm{ng} / \mathrm{mL})$ or plus hernandezine $(30 \mu \mathrm{M})$ for indicated time, relative ROS intensity (A) and NFkB activation (B) were tested. ${ }^{*} p<0.05$ vs. "C" group. ${ }^{*} p<0.05$ vs. LPS only group. ${ }^{* *} p<0.05$. Experiments in this figure were repeated three times, and similar results were obtained.
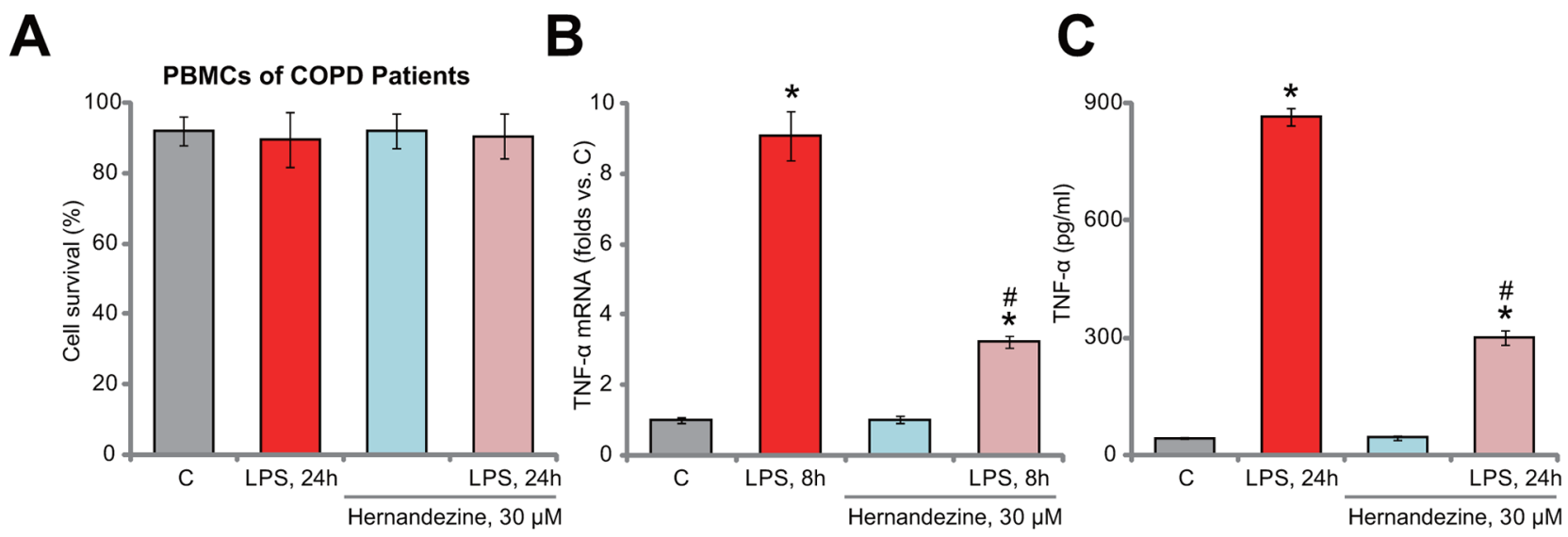

Figure 6: Hernandezine inhibits LPS-induced TNFa production in primary human PBMCs. Ex-vivo cultured PBMCs of COPD patients were treated with LPS $(100 \mathrm{ng} / \mathrm{mL})$ and/or hernandezine $(30 \mu \mathrm{M})$ for indicated time, cell survival was tested by trypan blue assay (A); relative TNF $\alpha$ mRNA expression ((B), qRT-PCR assay) and TNF $\alpha$ content in conditional medium ((C), ELISA assay) were also tested. ${ }^{*} p<0.05$ vs. "C" group. ${ }^{\#} p<0.05$ vs. LPS only group. Experiments in this figure were repeated three times, and similar results were obtained. 
blocked hernandezine-induced AMPK activation, and almost completely reversed its anti-LPS activity. Meanwhile, exogenous expression of caAMPK $\alpha$ also inhibited LPS-induced TNF $\alpha$ production. Importantly, hernandezine was almost invalid against LPS in the caAMPK $\alpha$-expressing cells. Thus, activation of AMPK by hernandezine is responsible for its anti-LPS activity in macrophage cells.

LPS, which is sensed by CD14 and LPS-binding protein, binds to Toll-like receptor 4 (TLR-4) on macrophages/monocytes [26, 27], which will activate downstream NFאB signaling to initiate pro-inflammatory response [26, 27]. ROS production is known to be critical in the process. Recent studies [19, 28, 29] including ours [11] have implied that AMPK could be effective in suppressing oxidative stresses. For example, energy depletion-activated AMPK-ACC signaling was shown to increase intracellular nicotinamide adenine dinucleotide phosphate (NADPH) content and to inhibit oxidative stress [28]. She et al., demonstrated that activation of AMPK significantly suppressed $\mathrm{H}_{2} \mathrm{O}_{2}$-induced oxidative damages [29]. Similarly, AMPK activation by cordycepin inhibited LPS-induced ROS accumulation [19]. Our previous studies have shown that GSK621 [10] or miR135b-5p [11] inhibited LPS-induced ROS production, thus blocking the downstream NFKB activation. This could be the key mechanism responsible for AMPK-induced antiLPS activity.

In line with these findings, we show that hernandezine largely inhibited LPS-induced ROS production and NFKB activation in U937 cells. AMPK inhibition, by targeted shRNA or dominant negative mutation, almost completely reversed hernandezine's above actions. Thus, we conclude that hernandezine activates AMPK signaling to inhibit LPS-induced ROS production and subsequent $\mathrm{NF} \kappa \mathrm{B}$ activation, which then leads to decreased TNF $\alpha$ mRNA synthesis and production. The detailed mechanism may warrant further investigations.

\section{MATERIALS AND METHODS}

\section{Chemicals and antibodies}

Hernandezine was purchased from EFE-Bio Company (Shanghai, China). LPS and puromycin were provided from Sigma Chemicals (Shanghai, China). The antibodies were all obtained from Cell Signaling Technology (Danvers, MA). Cell culture reagents were provided by Hyclone (Shanghai, China).

\section{Cell culture}

As described previously [11], the two human macrophage cell lines, U937 and THP-1, were cultured in
RPMI 1640 medium supplemented with 10\% FBS and 1\% glutamine at $37^{\circ} \mathrm{C}$.

\section{Ex-vivo culture of human PBMCs}

As described previously [9, 10], PBMCs of COPD patients (administrated at the Second Affiliated Hospital of Xi'an Jiao Tong University, Xi'an, China) were collected via lymphocyte separation medium (Sigma, Shanghai, China). The resulting PBMCs were cultured in DMEM plus $10 \%$ FBS, and necessary supplements [30]. Experiments and protocols requiring human samples were approved by the Ethics Committee and Internal Review Board of Xi'an Jiao Tong University. Written-informed consent was provided by each patient.

\section{Real-time PCR assay}

The detailed protocol for real-time reverse transcriptase quantitative polymerase chain reaction (qRTPCR) assay was described in previous studies [9-11]. The primers for TNF $\alpha$ mRNA and GAPDH mRNA were described previously [11]. All primers were synthesized by Genepharm (Shanghai, China). We utilized the comparative $\mathrm{Ct}\left(2^{-\Delta \Delta \mathrm{Ct}}\right)$ method to calculate relative $T N F \alpha$ mRNA expression [31-33]. GAPDH was always tested as the reference gene [33].

\section{TNF $\alpha$ enzyme-linked immunosorbent assay (ELISA) assay}

$\mathrm{TNF} \alpha$ protein content in the conditional medium was tested by the TNF $\alpha$ ELISA kit (R\&D Systems, Abingdon, UK), and detailed protocol was described previously [9].

\section{Western blotting assay}

As described [9-11], after applied treatment, cells were lysed, the protein lysates (20 $\mu \mathrm{g}$ per sample) were separated by the SDS-PAGE gel (10-12\%). Protein samples were then transferred onto PVDF membranes, which were then probed with indicated primary and corresponding secondary antibodies. The indicated bands were then visualized by the enhanced chemiluminescence (ECL, Amersham, Shanghai, China) regents [9].

\section{AMPKo $\operatorname{shRNA}$}

The two lentiviral human AMPK $\alpha$ short hairpin RNAs (shRNAs, "No1" and "No2", with non-overlapping sequences) were described previously [10, 11, 34]. Cells were incubated with the lentiviral shRNA for 24 hours, and were selected by puromycin $(1.0 \mu \mathrm{g} / \mathrm{mL})$ for another 12 days $[10,11,34]$. Western blotting assay was applied to confirm the stable knockdown of AMPK $\alpha$. The non-sense 
lentiviral control shRNA (Santa Cruz Biotech) was added to the control cells.

\section{AMPKa mutation}

The pSuper-puro construct with dominant negative AMPK $\alpha$ (T172A), the constitutively-active AMPK $\alpha$ (T172D), and the empty vector were provided by Dr. Lu's group [10, 11, 34]. We utilized Lipofectamine 2000 to transfect the mutant AMPK $\alpha$ or the empty vector to U937 cells. Stable cells were again selected by puromycin.

\section{ROS assay}

The detailed protocol for ROS assay was described previously $[10,11,19]$. Briefly, ROS content in cells with applied treatment was measured by dichlorofluorescin (DCF) oxidation assay. Cells were incubated with 10 $\mu \mathrm{M}$ of DCFH-DA (Invitrogen, Shanghai, China) for 30 $\mathrm{min}$, and were then washed in PBS for three times. DCF fluorescence intensity was then tested $[10,11,19]$.

\section{Measuring NFkB (p65) DNA-binding activity}

NFkB (p65) DNA-binding activity was tested as described in our previous studies [9-11]. Briefly, after applied treatment, $1.0 \mu \mathrm{g}$ of cell nuclear extracts per treatment were analyzed of the NFkB (p65) DNA-binding activity, via the TransAM ${ }^{\mathrm{TM}}$ ELISA kit (Active Motif, Carlsbad, CA) according to the recommended protocol. The OD value of treatment group was normalized to that of control group to reflect relative NFKB activity.

\section{Statistics analysis}

The statistical analyses were performed via the SPSS software (18.0), with $p<0.05$ taken as significant. Data were expressed as mean \pm standard deviation (SD). For comparisons among multiple groups, two-way ANOVA with the Bonferroni post hoc testing was performed.

\section{Author contributions}

All authors carried out the experiments, participated in the design of the study and performed the statistical analysis, conceived of the study, and participated in its design and coordination and helped to draft the manuscript. All authors read and approved the final manuscript.

\section{ACKNOWLEDGMENTS}

This work is partly supported by the NSFC.

\section{CONFLICTS OF INTEREST}

The authors declare no conflicts of interest.

\section{REFERENCES}

1. Barnes PJ. New anti-inflammatory targets for chronic obstructive pulmonary disease. Nat Rev Drug Discov. 2013; 12:543-559.

2. Brusasco V, Martinez F. Chronic obstructive pulmonary disease. Compr Physiol. 2014; 4:1-31.

3. Roversi S, Roversi P, Spadafora G, Rossi R, Fabbri LM. Coronary artery disease concomitant with chronic obstructive pulmonary disease. Eur J Clin Invest. 2014; 44:93-102.

4. Cosio MG, Saetta M, Agusti A. Immunologic aspects of chronic obstructive pulmonary disease. N Engl J Med. 2009; 360:2445-2454.

5. Lamela J, Vega F. Immunologic aspects of chronic obstructive pulmonary disease. N Engl J Med. 2009; 361:1024.

6. Rabinovich RA, Figueras M, Ardite E, Carbo N, Troosters T, Filella X, Barbera JA, Fernandez-Checa JC, Argiles JM, Roca J. Increased tumour necrosis factor-alpha plasma levels during moderate-intensity exercise in COPD patients. Eur Respir J. 2003; 21:789-794.

7. Profita M, Chiappara G, Mirabella F, Di Giorgi R, Chimenti L, Costanzo G, Riccobono L, Bellia V, Bousquet J, Vignola AM. Effect of cilomilast (Ariflo) on TNF-alpha, IL-8, and GM-CSF release by airway cells of patients with COPD. Thorax. 2003; 58:573-579.

8. Ouagued M, Martin-Chouly CA, Brinchault G, LeportierComoy C, Depince A, Bertrand C, Lagente V, Belleguic C, Pruniaux MP. The novel phosphodiesterase 4 inhibitor, CI-1044, inhibits LPS-induced TNF-alpha production in whole blood from COPD patients. Pulm Pharmacol Ther. 2005; 18:49-54.

9. Li P, Wu Y, Li M, Qiu X, Bai X, Zhao X. AS-703026 inhibits LPS-induced TNF $\alpha$ production through MEK/ERK dependent and independent mechanisms. PLoS One. 2015; 10:e137107.

10. Wu YH, Li Q, Li P, Liu B. GSK621 activates AMPK signaling to inhibit LPS-induced TNF $\alpha$ production. Biochem Biophys Res Commun. 2016; 480:289-295.

11. Li P, Fan JB, Gao Y, Zhang M, Zhang L, Yang N, Zhao X. miR-135b-5p inhibits LPS-induced TNF $\alpha$ production via silencing AMPK phosphatase Ppm1e. Oncotarget. 2016; 7:77978-77986. doi: 10.18632/oncotarget.12866.

12. Hardie DG, Ross FA, Hawley SA. AMPK: a nutrient and energy sensor that maintains energy homeostasis. Nat Rev Mol Cell Biol. 2012; 13:251-262.

13. Fan JB, Ruan JW, Liu W, Zhu LQ, Zhu XH, Yi H, Cui SY, Zhao JN, Cui ZM. miR-135b expression downregulates Ppm1e to activate AMPK signaling and protect osteoblastic cells from dexamethasone. Oncotarget. 2016; 7:7061370622. doi: 10.18632/oncotarget. 12138 .

14. Tang J, Feng Y, Tsao S, Wang N, Curtain R, Wang Y. Berberine and Coptidis rhizoma as novel antineoplastic 
agents: a review of traditional use and biomedical investigations. J Ethnopharmacol. 2009; 126:5-17.

15. Shen J, Liang L, Wang C. Perifosine inhibits lipopolysaccharide (LPS)-induced tumor necrosis factor (TNF)-alpha production via regulation multiple signaling pathways: new implication for Kawasaki disease (KD) treatment. Biochem Biophys Res Commun. 2013; 437:250-255.

16. Zhao X, Zmijewski JW, Lorne E, Liu G, Park YJ, Tsuruta Y, Abraham E. Activation of AMPK attenuates neutrophil proinflammatory activity and decreases the severity of acute lung injury. Am J Physiol Lung Cell Mol Physiol. 2008; 295:L497-504.

17. Cacicedo JM, Yagihashi N, Keaney JF Jr, Ruderman NB, Ido Y. AMPK inhibits fatty acid-induced increases in NF-kappaB transactivation in cultured human umbilical vein endothelial cells. Biochem Biophys Res Commun. 2004; 324:1204-1209.

18. Ducommun S, Ford RJ, Bultot L, Deak M, Bertrand L, Kemp BE, Steinberg GR, Sakamoto K. Enhanced activation of cellular AMPK by dual-small molecule treatment: AICAR and A769662. Am J Physiol Endocrinol Metab. 2014; 306:E688-696.

19. Zhang JL, $\mathrm{Xu}$ Y, Shen J. Cordycepin inhibits lipopolysaccharide (LPS)-induced tumor necrosis factor (TNF)-alpha production via activating amp-activated protein kinase (AMPK) signaling. Int J Mol Sci. 2014; 15:12119-12134.

20. Law BY, Mok SW, Chan WK, Xu SW, Wu AG, Yao XJ, Wang JR, Liu L, Wong VK. Hernandezine, a novel AMPK activator induces autophagic cell death in drug-resistant cancers. Oncotarget. 2016; 7:8090-8104. doi: 10.18632/ oncotarget.6980.

21. Hardie DG. AMP-activated/SNF1 protein kinases: conserved guardians of cellular energy. Nat Rev Mol Cell Biol. 2007; 8:774-785.

22. Jiang H, Liu W, Zhan SK, Pan YX, Bian LG, Sun B, Sun QF, Pan SJ. GSK621 targets glioma cells via activating AMP-activated protein kinase signalings. PLoS One. 2016; 11:e0161017.

23. Guo S, Mao L, Ji F, Wang S, Xie Y, Fei H, Wang XD. Activating AMP-activated protein kinase by an alpha1 selective activator compound 13 attenuates dexamethasoneinduced osteoblast cell death. Biochem Biophys Res Commun. 2016; 471:545-552.
24. Chen L, Chen Q, Deng G, Kuang S, Lian J, Wang M, Zhu H. AMPK activation by GSK621 inhibits human melanoma cells in vitro and in vivo. Biochem Biophys Res Commun. 2016; 480:515-521.

25. Sujobert P, Poulain L, Paubelle E, Zylbersztejn F, Grenier A, Lambert M, Townsend EC, Brusq JM, Nicodeme E, Decrooqc J, Nepstad I, Green AS, Mondesir J, et al. Co-activation of AMPK and mTORC1 induces cytotoxicity in acute myeloid leukemia. Cell Rep. 2015; 11:1446-1457.

26. Bryant CE, Spring DR, Gangloff M, Gay NJ. The molecular basis of the host response to lipopolysaccharide. Nat Rev Microbiol. 2010; 8:8-14.

27. Miller SI, Ernst RK, Bader MW. LPS, TLR4 and infectious disease diversity. Nat Rev Microbiol. 2005; 3:36-46.

28. Jeon SM, Chandel NS, Hay N. AMPK regulates NADPH homeostasis to promote tumour cell survival during energy stress. Nature. 2012; 485:661-665.

29. She C, Zhu LQ, Zhen YF, Wang XD, Dong QR. Activation of AMPK protects against hydrogen peroxide-induced osteoblast apoptosis through autophagy induction and NADPH maintenance: new implications for osteonecrosis treatment? Cell Signal. 2014; 26:1-8.

30. Du SL, Yuan X, Zhan S, Tang LJ, Tong CY. Trametinib, a novel MEK kinase inhibitor, suppresses lipopolysaccharideinduced tumor necrosis factor (TNF)-alpha production and endotoxin shock. Biochem Biophys Res Commun. 2015; 458:667-673.

31. Livak KJ, Schmittgen TD. Analysis of relative gene expression data using real-time quantitative PCR and the (2- $\Delta \Delta \mathrm{CT})$ method. Methods. 2001; 25:402-408.

32. Li ZW, Cai S, Liu Y, Yang CL, Tian Y, Chen G, Cao C. Over-expression of Galphai3 in human glioma is required for Akt-mTOR activation and cell growth. Oncotarget. 2016 Aug 1. doi: 10.18632/oncotarget.10995. [Epub ahead of print].

33. Kwon MY, Park E, Lee SJ, Chung SW. Heme oxygenase-1 accelerates erastin-induced ferroptotic cell death. Oncotarget. 2015; 6:24393-24403. doi: 10.18632/ oncotarget.5162.

34. Lu PH, Chen MB, Ji C, Li WT, Wei MX, Wu MH. Aqueous Oldenlandia diffusa extracts inhibits colorectal cancer cells via activating AMP-activated protein kinase signalings. Oncotarget. 2016; 7:45889-45900. doi: 10.18632/ oncotarget.9969. 